\title{
THE APPLICABILITY OF THE MASS-FLOW-MODEL ACCORDING TO ISO 6358 WITH THE PARAMETER CRITICAL CONDUCTANCE C AND CRITICAL PRESSURE RATIO B FOR GASES IN HIGH-PRESSURE RANGE UP TO 300 BAR
}

\author{
Lucian Pasieka* \\ Eugen Seitz AG, Spitalstrasse 204, 8623 Wetzikon, Switzerland \\ *Corresponding author: Tel.: +41 4493181 18; E-mail address: lpasieka@seitz.ch
}

\begin{abstract}
Mass flow models for gaseous media describe the relationship between gas flow through throttle elements depending on pressure, temperature and type of medium. These models are used to calculate pneumatic components, to simulate pneumatic systems or to plan facilities. The known flow models are verified for ideal gases, such those as according to ISO 6358-1 (International Standard ISO 63581: pneumatic fluid power - Determination of flow-rate characteristics of components using compressible fluids: General rules and test methods for steady-state flow, 2013). But they fail with real gases, especially at higher pressures. The objective of this contribution is to recommend a simple-touse model for the mass flow calculation of real gases. The advantage of the $\mathrm{C}$ and $\mathrm{b}$ model is the use of only two parameters. A testbench for high pressure up to 300 bar was used for model verification with air and hydrogen. The Library for standard dry air and hydrogen up to 1000 bar was used for the state variables of the real gases. The parameters $C$ and $b$ are obtained, applied and verified in the state space of real gas. With the $\mathrm{C}$ and $\mathrm{b}$ parameter it is possible to simulate not only the single component (valve, throttle, nozzle and so on) but also the whole system with a complete system simulation, like such as hydrogen filling stations.
\end{abstract}

Keywords: mass flow model, real gas

\section{INTRODUCTION}

In the planning of facilities for gases the components such as valves and throttle valves must be considered as flow resistances. With a view to a system simulation, it is desirable to have the mass flow characteristics of all individual components to know the states and the operating behaviour of the entire system in a system simulation for the purpose of calculation. In the development of components, the goal is to identify the mass flow characteristic of the component concerned to make the selection easier for the user. In the 90 s different methods of measurements have been compared in relation to their suitability for the simulation of pneumatic systems [2].

The proposed standard ISO 6358 "Pneumatic fluid power - Components using compressible fluids - Determination of flow-rate characteristics (1989)" [3] has become proved useful and a recognized standard in practice. This first edition of ISO 6358-1 (2013) [12], cancels and replaces ISO 6358:1989, which has been technically revised. This standard defines a method for measurement and the determination of fluid power parameters for components of pneumatics, such as valves. With the aid of the parameters, the mass flow in the stationary state for any pressure ratio under the assumption of the ideal gases are calculated. We use the alternative test method ISO 6358-1, Annex A, page 20. This method complies with ISO 6358 (1989) with the addition of using stagnation pressure instead of static pressure.

The advantage of the mass flow model according to ISO 6358-1 lies in the ease of handling, since only two parameters $\mathrm{C}$ and $\mathrm{b}$ are needed. Series and parallel circuits of components can be calculated, see [8]. All losses that occur as a pressure drop across a component are recorded using the two parameters. At this point, the question arises: what are the special features of mass flow in the high-pressure range? 
For example, the design of safety valves in the high-pressure range based on models used up to 3000 bar according to [1] equations for ideal gases. Schmidt et al. [10] shows that there is no suitable mass flow model that can be used in the real gas range.

In ISO 6358-1 there are no application limits for the pressure range specified. The mass flow model is based on the assumptions of the ideal gas and, therefore, has been validated at low pressure applications up to 16 bar. The purpose of this study is to evaluate the proposed mass flow model of ISO 6358-1 in real gas applications and to verify the model based on measurements up to 300bar. Attention is focused on the nonlinear flow characteristics in the subcritical flow range, due larger deviations in this range compared to the use of the model simplification.

\section{BASICS OF THE MASS FLOW MODEL}

The basis for the mass flow model is the stationary flow through a nozzle with the following assumptions:

- frictionless and adiabatic flow (= isentropic)

- nozzle with rounded contour

- no technical work is done and

- the potential energy of the gas can be neglected, see Fig. 1

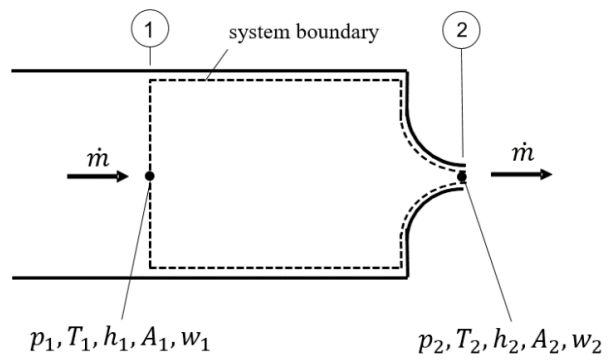

Figure 1: Schematic representation of the system boundary of a throttle element for the derivation of the mass flow equation as well as the specific heat capacities

With the help of the first law for stationary flow processes

$$
\mathrm{h}_{1}+\frac{\mathrm{w}_{1}^{2}}{2}=\mathrm{h}_{2}+\frac{\mathrm{w}_{2}^{2}}{2}
$$

With the assumption of a large volume in front of the narrowest cross-section, it can be assumed that $w_{l} \approx 0$. Consequently

$$
\frac{w_{2}^{2}}{2}=h_{1}-h_{2}=c_{p}\left(T_{1}-T_{2}\right)
$$

With the ideal gas law

$$
\begin{aligned}
& T_{1}=\frac{p_{1}}{R \cdot \rho_{1}} \\
& c_{p}-c_{v}=R \\
& \frac{c_{p}}{c_{v}}=\kappa \\
& \frac{c_{p}}{R}=\frac{\kappa}{\kappa-1}
\end{aligned}
$$

and the equation for an isentropic state change

$$
\frac{T_{2}}{T_{1}}=\left(\frac{p_{2}}{p_{1}}\right)^{\frac{\kappa-1}{\kappa}}
$$

follows

$$
w_{2}=\sqrt{2 \frac{\kappa}{\kappa-1} \frac{p_{1}}{\rho_{1}}\left[1-\left(\frac{p_{2}}{p_{1}}\right)^{\frac{\kappa-1}{\kappa}}\right]}
$$

By using the continuity equation

$$
\begin{aligned}
\dot{m} & =w_{2} A_{2} \rho_{2} \\
\frac{\rho_{2}}{\rho_{1}} & =\left(\frac{p_{2}}{p_{1}}\right)^{\frac{1}{\kappa}}
\end{aligned}
$$

one obtains (11) for the calculation of the mass flow [8]

$\dot{m}=A_{2} \sqrt{2 p_{1} \rho_{1}} \sqrt{\frac{\kappa}{\kappa-1}\left[\left(\frac{p_{2}}{p_{1}}\right)^{\frac{2}{\kappa}}-\left(\frac{p_{2}}{p_{1}}\right)^{\frac{\kappa+1}{\kappa}}\right]}$

The assumptions don't include flow-reducing effects, so it's necessary to introduce two geometry-dependent effects of beam constriction and fluid friction are introduced, see (12). The jet entanglement is the reason that due to the inertia and the nozzle contour not the entire geometric flow area is available for the flowing fluid. This is done with the beam constriction number $\alpha$, which is always less than $1(\alpha<1)$. The fluid friction leads to flow losses. The so-called speed digit $\varphi$ is considered to be $0<\varphi<1$. This results in the following mass flow equation

$$
\dot{m}=\alpha \varphi A_{2} \Psi \sqrt{2 p_{1} \rho_{1}} \text {. }
$$


The right root expression in (11) describes the outflow function $\Psi$, see Fig. 2

$$
\Psi=\sqrt{\frac{\kappa}{\kappa-1}\left[\left(\frac{p_{2}}{p_{1}}\right)^{\frac{2}{\kappa}}-\left(\frac{p_{2}}{p_{1}}\right)^{\frac{\kappa+1}{\kappa}}\right]}
$$

The flow function $\Psi$ has a maximum that characterizes the transition between the supercritical and subcritical flow range. The pressure ratio at which the maximum occurs, is called critical pressure ratio $b$ because there is speed of sound in the most-narrow cross-section. It results from building the 1st derivative of the outflow function with the respect to the pressure ratio.

$$
\frac{d \Psi}{d\left(\frac{p_{2}}{p_{1}}\right)}=\Psi^{\prime}=0
$$

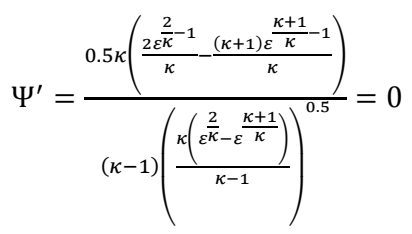

with $\frac{p_{2}}{p_{1}}=\varepsilon$

According to equation (15), the derivation can be reduced to the internal function. This means

$$
\begin{aligned}
& 0=\frac{2}{\kappa}(\varepsilon)^{\frac{2-\kappa}{\kappa}}-\frac{\kappa+1}{\kappa}(\varepsilon)^{\frac{1}{\kappa}} \\
& \frac{2}{\kappa+1}=(\varepsilon)^{\frac{\kappa-1}{\kappa}} \\
& b=\left(\frac{p_{2}}{p_{1}}\right)_{\text {crit }}=\left(\frac{2}{\kappa+1}\right)^{\frac{\kappa}{\kappa-1}}
\end{aligned}
$$

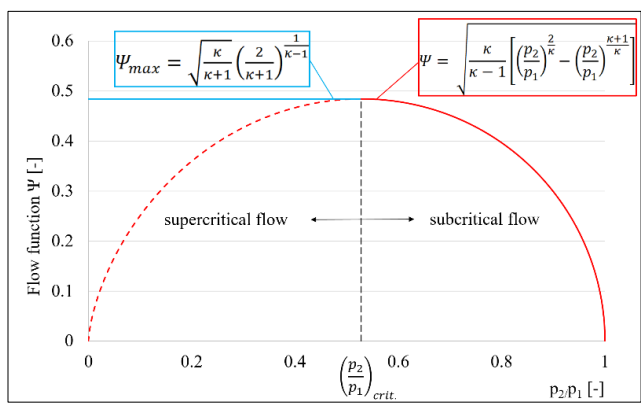

Figure 2: Flow function $\Psi$ via the pressure ratio $\mathrm{p}_{2} / \mathrm{p}_{1}$
The maximum of the discharge function $\Psi_{\max }$ is obtained by using the critical pressure ratio $b$ (18) in the discharge function (13).

$$
\Psi_{\text {max }}=\sqrt{\frac{\kappa}{\kappa+1}}\left(\frac{2}{\kappa+1}\right)^{\frac{1}{\kappa-1}}
$$

The fact that $\Psi_{\max }$ is a high point can be shown by forming the 2 nd derivative starting from (15) and using the critical pressure ratio $b$ from (18).

After evaluating it can be shown that $\kappa>1$ applies to all: $\Psi "<0$ i.e. it is a maximum, see [11].

With the view to the mass flow model for ideal gases, (12), the outflow function $\Psi$ is divided into two areas. In the subcritical area, the outflow function is (13), see Fig. 2. At the critical pressure ratio $b$ the medium flows at the speed of sound through the nozzle. If the pressure ratio is reduced $\mathrm{p}_{2} / \mathrm{p}_{1}<\mathrm{b}$ (by reducing the outlet pressure $\mathrm{p}_{2}$ ) the flow velocity does not increase any further, as this is limited by the speed of sound. As a result, the mass flow stagnates in the supercritical range at its maximum value, see blue line in Fig. 2. is used in the calculation of the mass flow through the maximum considered outflow $\Psi_{\max }$ (12).

With the generally valid mass flow equation (12) the mass flow thought throttle elements can be described analytically. The disadvantage of equation (12) is that the individual coefficients can be determined only with great effort. That's why there were different proposals, the generally valid mass flow equation with an approximation function that can be described by a few measurable parameters. Advantageous in this procedure is that the critical pressure ratio $b$ is not a constant value (18) but assumes values as they occur in practice. The critical pressure ratio depends both on the medium and the flowreducing effects and generally ranges between $0,2 \ldots 0,54$. According to a proposal by Sanville [9], the outflow function $\Psi$ (13), can be described using an elliptical approximation (20).

$$
\Psi=\sqrt{\frac{\kappa}{\kappa-1}\left[\left(\frac{p_{2}}{p_{1}}\right)^{\frac{2}{\kappa}}-\left(\frac{p_{2}}{p_{1}}\right)^{\frac{\kappa+1}{\kappa}}\right]} \approx \Psi_{\max } \sqrt{1-\left(\frac{\frac{p_{2}}{p_{1}}-b}{1-b}\right)^{2}}
$$

Further approximations of the outflow function $\Psi$ according to [9] are presented in [11] for comparison. 
Because the approximation (20) has the smallest relative error of $0.30 \%$ compared to (13), it was included as a basis in ISO 6358. This model contains two parameters: The critical pressure ratio $\mathrm{b}$ and the critical conductance $\mathrm{C}$, see (21) and (22). The critical conductance $C$ includes the maximum of flow function $\Psi_{\max }$, as well as the cross-sectional area $\mathrm{A}_{2}$ and the flowreducing effects (fluid friction, wall friction, beam constriction, deflections, compaction surge losses), see (12). With the ratio $\left(\mathrm{T}_{0} / \mathrm{T}_{1}\right)^{0.5}$ the mass flow model was determined independently of the temperature at which $\mathrm{C}$ was measured. The mass flow model measured at temperature $\mathrm{T}_{1}$, conductance $\mathrm{C}$ is related to temperature $\mathrm{T}_{0}$, see (23), in order to be able to calculate the mass flow at any temperature $T_{1}$. Basically, this relationship is only valid for ideal gas.

$$
\begin{aligned}
& \dot{m}=p_{1} \cdot C \cdot \varrho_{0} \cdot \sqrt{\frac{T_{0}}{T_{1}}} \sqrt{1-\left(\frac{\frac{p_{2}}{p_{1}}}{1-b}\right)^{2}} \text { for } \frac{p_{2}}{p_{1}}>b \\
& \dot{m}=p_{1} \cdot C \cdot \varrho_{0} \cdot \sqrt{\frac{T_{0}}{T_{1}}} \quad \text { for } \frac{p_{2}}{p_{1}} \leq b
\end{aligned}
$$

\section{DETERMINATION OF THE MODEL PARAMETERS ACCORDING TO ISO 6358}

The procedure for determining the model parameters $\mathrm{C}$ and $\mathrm{b}$ is described in detail in ISO 6358-1, Annex A [12]. This requires five individual measurements under steady-state conditions. The measured values which are shown in Table 1 as example. The critical conductance $C_{L P}$ is determined at the supercritical measuring point marked with *. The influence of stagnation pressure (see [12] p22, A.1) has been checked and can be neglected because the diameter of the pressure measuring tube is large enough. Using the measuring points $j=1 \ldots 4$ in the subcritical flow area, the critical pressure ratio
$b_{L P}$ is calculated as the arithmetic mean value. The parameters $C_{L P}$ and $b_{L P}$ are stored in the three right-hand columns of Table 1 the calculated and measured mass flow values are compared. The averaged relative deviation between mass flow model and measured values in this example is $3.2 \%$.

In section 2 the basics of the mass flow model for ideal gases according to ISO 6358 were shown and illustrated with an example, Table 1. The following experimental investigations are aimed at examining the validity of the mass flow model in the real gas range. For this goal, experiments with air in the pressure range up to $300 \mathrm{bar}$ and at a temperature of $20^{\circ} \mathrm{C}$ were carried out. Under these conditions, air has a real gas factor $\mathrm{Z}_{\text {Air }}=1.105$.

\section{EXPERIMENTAL INVESTIGATION IN THE HIGH-PRESSURE RANGE}

\subsection{Determination of the model parameters in the high-pressure range}

In the following tests the parameters $\mathrm{C}$ and $\mathrm{b}$ are determined at $p_{1}=300$ bar. These are referred to as high pressure $\mathrm{C}_{\mathrm{HP}}$ and $\mathrm{b}_{\mathrm{HP}}$. The procedure is identical except for the pressure as in the example in section 3 in the low-pressure range. The pneumatic conductance $\mathrm{C}_{\mathrm{HP}}$ was measured at supercritical flow identified by the following equation

$$
C_{H P}=\frac{\dot{m}^{*}}{\varrho_{0} p_{1}^{*}} \sqrt{\frac{T_{1}^{*}}{T_{0}}}
$$

The measuring points for identifying the critical pressure ratio $b$, see $j=1 \ldots 4$ in Table 2, are in the subcritical flow range of the throttle at $80,60,40$ and $20 \%$ of the supercritical mass flow $\dot{\mathrm{m}}^{*}$. At each measuring point a critical pressure ratio bj can be determined. The critical pressure ratio $b$ of the nozzle element results from the arithmetic mean of these values, see (24), and

Table 1: Parameters ISO 6358-1

\begin{tabular}{|l|l|l|l|l|l|l|l|l|l|l|}
\hline $\begin{array}{l}\mathrm{j} \\
{[-]}\end{array}$ & $\begin{array}{l}\mathrm{p}_{1} \\
\mathrm{~Pa}\end{array}$ & $\begin{array}{l}\mathrm{p}_{2} \\
\mathrm{~Pa}\end{array}$ & $\begin{array}{l}\mathrm{p}_{2} / \mathrm{p}_{1} \\
{[-]}\end{array}$ & $\begin{array}{l}\mathrm{T}_{1} \\
{[\mathrm{~K}]}\end{array}$ & $\begin{array}{l}\mathrm{b}_{\mathrm{j}} \\
{[-]}\end{array}$ & $\begin{array}{l}\mathrm{C}_{\mathrm{LP}} \\
{[\mathrm{s} \mathrm{m} / \mathrm{kg}]}\end{array}$ & $\begin{array}{l}\mathrm{b}_{\mathrm{LP}} \\
{[-]}\end{array}$ & $\begin{array}{l}\dot{m}_{\text {calc }} \\
{[\mathrm{g} / \mathrm{s}]}\end{array}$ & $\begin{array}{l}\dot{m}_{\text {meas }} \\
{[\mathrm{g} / \mathrm{s}]}\end{array}$ & $\begin{array}{l}\Delta \dot{m} / \dot{m} \cdot 10^{2} \\
{[\%]}\end{array}$ \\
\hline$*$ & 995216 & 102586 & 0,103 & 291,6 & & $4,06 \mathrm{E}-10$ & & 0,481 & 0,481 & 0,00 \\
\hline 1 & 995349 & 827861 & 0,832 & 291,7 & 0,539 & & & 0,358 & 0,372 & $-3,58$ \\
\hline 2 & 995825 & 892146 & 0,896 & 291,7 & 0,521 & & 0,494 & 0,293 & 0,300 & $-2,41$ \\
\hline 3 & 996358 & 959180 & 0,963 & 291,7 & 0,488 & & & 0,181 & 0,180 & 0,57 \\
\hline 4 & 997381 & 983959 & 0,987 & 291,7 & 0,428 & & & 0,110 & 0,104 & 6,23 \\
\hline
\end{tabular}


indicates at which pressure ratio $\mathrm{p}_{2} / \mathrm{p}_{1}$ sound velocity in the narrowest cross-section prevails.

$b_{H P}=\frac{1}{n} \sum_{j=1}^{n}\left(1-\frac{\frac{p_{1, j}-p_{2, j}}{p_{1, i}}}{1-\sqrt{1-\left(\frac{\dot{m}_{i}}{C_{H P \varrho_{0} p_{1, j}}} \sqrt{\frac{T_{1, j}}{T_{0}}}\right)^{2}}}\right)$

\subsection{Verification of the parameters $C_{H P}$ and bHP in High pressure range}

To validate the parameters $\mathrm{C}_{\mathrm{HP}}$ and $\mathrm{b}_{\mathrm{HP}}$, for 10 to $90 \%$ of the critical mass flow in $20 \%$ steps measurements are carried out, see $\mathrm{j}=5$...9 in Table 2. The investigation was made with two flow geometries. The "valve" geometry shown in Figure 3 in comparison to the "nozzle" nominal diameter (NW) $0.5 \mathrm{~mm}$ " has more cross-sectional changes and deflections. The mass flow function (25) and (26) with parameters $\mathrm{C}_{\mathrm{HP}}$ and $\mathrm{b}_{\mathrm{HP}}$ are measured in [11] and are valid for an inlet pressure $\mathrm{p}_{1}=300$ bar.

$\dot{m}=p_{1} \cdot C_{H P} \cdot \varrho_{0} \cdot \sqrt{\frac{T_{0}}{T_{1}}} \sqrt{1-\left(\frac{\frac{p}{2}_{1}-b_{H P}}{1-b_{H P}}\right)^{2}}$ for $\frac{p_{2}}{p_{1}}>b$

$\dot{m}=p_{1} \cdot C_{H P} \cdot \varrho_{0} \cdot \sqrt{\frac{T_{0}}{T_{1}}} \quad$ for $\frac{p_{2}}{p_{1}} \leq b$

A check of the model is only in the subcritical flow range $\mathrm{p}_{2} / \mathrm{p}_{1}>\mathrm{b}_{\mathrm{HP}}$ necessary, since the flow in the supercritical range is only limited by the local speed of sound. At a further reduction of the pressure ratio $\mathrm{p}_{2} / \mathrm{p}_{1}$ the state variables determining the mass flow are constant, which also makes the conductance $\mathrm{C}_{\mathrm{HP}}$ a constant variable.

The relative deviation between the calculated mass flow with the ellipse model and the measured mass flow is between -1.2 and $+4.4 \%$. The maximum measuring error of the Coriolis mass flow sensor is according to the manufacturer's specifications (Endress and Hauser) $0.10 \%$ of the measured value.

The average of all 10 measuring points results in a relative mass flow deviation of $1.0 \%$. In both measurements the maximum deviation is when the pressure ratio $\mathrm{p}_{2} / \mathrm{p}_{1}$ is close to 1 . It is therefore to be expected that the relative deviation of the ellipse model continues to rise at $\mathrm{p}_{2} / \mathrm{p}_{1}>0.998$. In the remaining range of the pressure-mass flow characteristic a very good agreement can be established between model and reality.

For parameter determination at high pressure, the higher expenditure on pressure, temperature and flow measurement technology and an increased demand for media would be disadvantageous.

\section{APPLICATIONS OF THE PARAMETERS CLP AND BLP IN THE HIGH-PRESSURE RANGE}

The application of the $\mathrm{C}, \mathrm{b}$ model in high pressure leads to the question of the validity range of the determined parameters. Since in the state region of real gases the gas properties are not linearly dependent on pressure and temperature, it can be assumed that the low-pressure parameters are not easily applicable in the high-pressure range.

Table 2: Measured values for identification of $\mathrm{C}_{\mathrm{HP}}, \mathrm{b}_{\mathrm{HP}}$ at $\mathrm{p}_{1}=300$ bar at the geometry "orifice $\mathrm{NW} 0,5 \mathrm{~mm}$ "

\begin{tabular}{|l|l|l|l|l|l|l|l|l|l|l|l|l|}
\hline $\begin{array}{l}\mathrm{J} \\
{[-]}\end{array}$ & $\begin{array}{l}\mathrm{p}_{1} \\
\mathrm{bar}\end{array}$ & $\begin{array}{l}\mathrm{p}_{2} \\
\mathrm{bar}\end{array}$ & $\begin{array}{l}\mathrm{p}_{2} / \mathrm{p}_{1} \\
{[-]}\end{array}$ & $\begin{array}{c}\dot{m} \\
{[\mathrm{~g} / \mathrm{s}]}\end{array}$ & $\begin{array}{l}\mathrm{b}_{\mathrm{j}} \\
{[-]}\end{array}$ & $\begin{array}{l}\mathrm{C}_{\mathrm{HP}} \\
{\left[\mathrm{s} \mathrm{m}^{4} / \mathrm{kg}\right]}\end{array}$ & $\begin{array}{l}\mathrm{b}_{\mathrm{HP}} \\
{[-]}\end{array}$ & $\begin{array}{l}\mathrm{j} \\
{[-]}\end{array}$ & $\begin{array}{l}\mathrm{p}_{2} / \mathrm{p}_{1} \\
{[-]}\end{array}$ & $\begin{array}{l}\dot{m}_{\text {calc }} \\
{[\mathrm{g} / \mathrm{s}]}\end{array}$ & $\begin{array}{l}\dot{m}_{\text {meas }} \\
{[\mathrm{g} / \mathrm{s}]}\end{array}$ & $\begin{array}{l}\Delta \dot{m} / \\
\dot{m} \cdot 1 \\
0^{2}\end{array}$ \\
\hline$*$ & 296,77 & 9,94 & 0,033 & 16,02 & & $4,53 \mathrm{E}-10$ & & 5 & 0,695 & 14,44 & 14,49 & $-0,40$ \\
\hline 1 & 296,72 & 235,21 & 0,793 & 12,73 & 0,472 & & & 6 & 0.861 & 10,76 & 10,88 & $-1,17$ \\
\hline 2 & 296.48 & 265,70 & 0,896 & 9,58 & 0,478 & & 0,463 & 7 & 0,930 & 7,91 & 7,90 & 0,14 \\
\hline 3 & 297,21 & 282,41 & 0,950 & 6,70 & 0,456 & & & 8 & 0,978 & 4,57 & 4,52 & 1,05 \\
\hline 4 & 297,22 & 293,72 & 0,988 & 3,30 & 0,448 & & & 9 & 0,998 & 1,44 & 1,38 & 4,40 \\
\hline
\end{tabular}




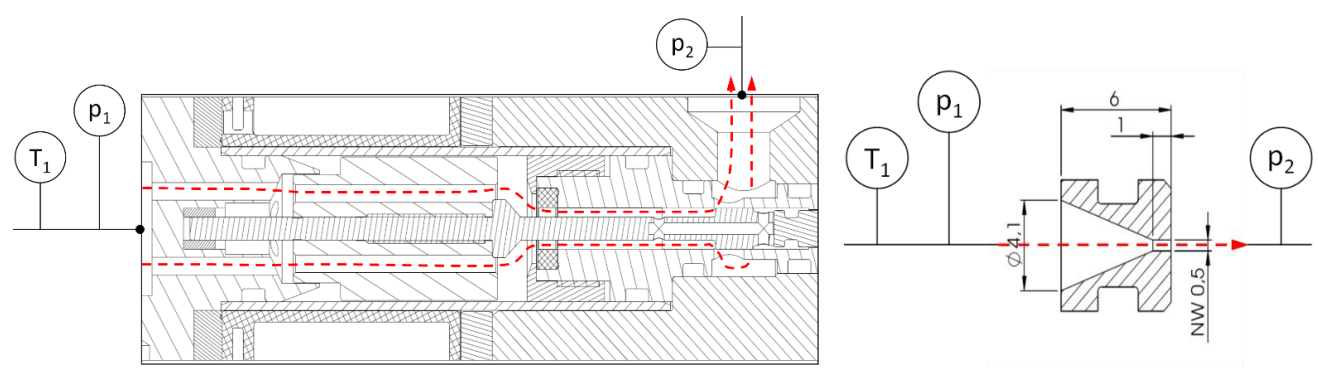

Figure 3: Illustration of the valve design (left) and the nozzle NW $0.5 \mathrm{~mm}$ (right).

The ellipse parameters $\mathrm{C}$ and $\mathrm{b}$ of the flow geometries "valve" and "nozzle NW $0.5 \mathrm{~mm}$ " were also determined in the ideal gas range according to ISO 6358. The comparison of the high- and low- pressure parameters in Fig. 4 shows that there is a "local validity" of the parameters in relation to the thermodynamic state. Strictly speaking, local validity means that the parameters $\mathrm{C}$ are only valid at the status point by which they were measured. The pneumatic conductance $\mathrm{C}_{\mathrm{HP}}$, which was determined at 300 bar, is at both geometries about $12 \%$ higher than at 10 bar, Fig. 4 . The associated increase in mass flow through a geometrically identical throttle is on the one hand influenced by the real gas behavior of the air, as well as from the changed flow-reducing effects in the high-pressure range. The critical pressure ratio $b_{H P}$ is reduced by about $9 \%$ versus the critical pressure ratio in the range of the ideal gas due to different thermodynamic state properties of the real gas.

The real gas properties lead to parameters that are only locally valid. Since the deviation of the pressure-flow characteristics is decisive for the user the parameters determined at high and low pressure were applied at 300 bar. The comparison of the two calculated mass flow functions is shown in Fig. 5 and 5. The deviation of the pneumatic conductivity leads to mass flow errors in the entire pressure range. As can be seen in

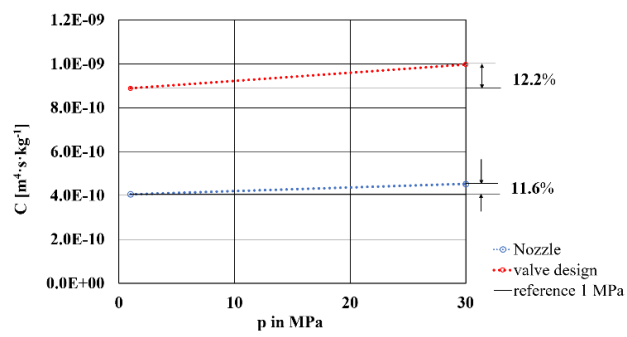

Figure 4: Comparison of measured conductance $\mathrm{C}$ of air in the ideal and real state range

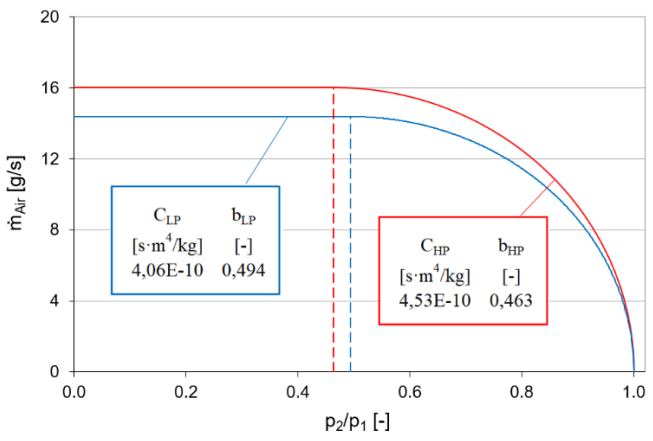

Figure 5: Comparison of the calculated mass flows with $\mathrm{C}_{\mathrm{LP}}, \mathrm{b}_{\mathrm{LP}}$ and $\mathrm{C}_{\mathrm{HP}}, \mathrm{b}_{\mathrm{HP}}$ at 300 bar. Geometry: "Nozzle" NW $0.5 \mathrm{~mm}$ conductance $\mathrm{C}$ affects the overpressure and subcritical flow. The deviation of the critical pressure ratio $b$ leads to comparatively lower mass flow errors than with the conductance, since the derivation of the ellipse function at critical pressure ratio becomes zero due to the definition. 


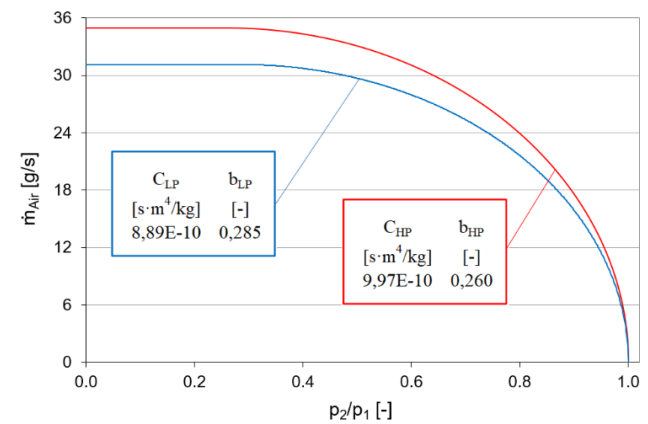

Figure 6: Comparison of the calculated mass flows with $C_{L P}, b_{L P}$ and $C_{H P}, b_{H P}$ at 300 bar. Geometry: "Valve"

\section{APPLICATION OF THE PARAMETERS CLP AND BLP IN THE HIGH-PRESSURE RANGE UNDER CONSIDERATION OF THE REAL GAS BEHAVIOUR IN THE NARROWEST CROSS-SECTION}

In the following, the influence of the real gas properties on the stationary mass flow through a throttle, considering pressure and temperature in the narrowest cross section is described. To calculate the change of state of a throttling of gaseous fluids, the system limit is chosen as shown in Fig. 1. The mass flow is limited by the thermodynamic state of the medium in the narrowest cross-section (state 2 in Fig. 1). The following assumptions were made to determine the condition of the medium in the narrowest cross-section:

- Real gas properties of dry air in the singlephase region according to Kretzschmar [5] based on the equation of state of Lemmon et al.

- The speed of sound prevails in the narrowest cross-section

- The media speed at the input is negligible

- $\mathrm{w}_{1}=0$

- Height differences are neglected

- No technical work is performed

- No heat is exchanged across the system

- boundaries $=>$ adiabatic

- No losses occur $=>$ reversible

- Adiabatic and reversible $=>$ isentropic

With the assumptions made, the thermodynamic state in the narrowest cross-section can be calculated iteratively and thus the influence of the real gas on the mass flow can be determined by the throttle. The schematic in Fig. 7 shows the sequence of calculation for the iterative calculation of the state variables.

The inlet pressure and the inlet temperature are constant in every iterative calculation. Exclusively for the starting point of the calculation it was assumed that ideal gas flows and an isentropic change of state is present. This results in the critical pressure ratio of the throttle $\left(b_{\text {crit, ideal }}=0.528\right)$, which means that the start pressure can be determined in the narrowest cross-section.

$$
p_{2, \text { real }}=p_{1, \text { real }} \cdot b_{\text {crit,ideal }} .
$$

Due to the pressure and temperature in the narrowest cross-section the thermodynamic initial state is defined. With the real isentropic exponent $\kappa_{2 \text {,real }}$ and the real gas factor $Z_{2 \text {,real the }}$ local isentropic sound velocity $\mathrm{w}_{2}$,real of the medium calculated

$w_{2, \text { real }}=\sqrt{\kappa_{2, \text { real }} \cdot T_{2, \text { real }} \cdot R \cdot Z_{2, \text { real }}}$

From the first law of thermodynamics follows for an adiabatic, stationary throttling without consideration of the height differences for the enthalpy in the narrowest cross-section

$h_{2, \text { real }}=h_{1, \text { real }}-\frac{w_{2, \text { real }}^{2}}{2}$.

The temperature of the first iteration step can be calculated from the enthalpy and the pressure at state 2 . We use the backward function $T=T(p, h)$, see [5]. With this temperature the next iteration loop starts, as shown in Fig. 7. The critical pressure ratio of the ideal gas is obtained from the derivative of the outflow function (13) according to the pressure ratio $\mathrm{p}_{2} / \mathrm{p}_{1}$.

In contrast to the critical pressure ratio of the nozzle under the conditions of the ideal gas in the starting step (18), in the further steps the critical pressure ratio is calculated as a function of the real isentropic exponent $\kappa_{2}$,real.

$\left(\frac{p_{2, \text { real }}}{p_{1, \text { real }}}\right)_{\text {crit,real }}=\left(\frac{2}{\kappa_{2, \text { real }}+1}\right)^{\frac{\kappa_{2, \text { real }}}{\kappa_{2, \text { real }}{ }^{-1}}}$

Since the real gas properties are strongly dependent on the medium, the iterative calculation for air and hydrogen is carried out. The real gas properties of Normal hydrogen in the single-phase region based on equation of state from Leachman et al. [7] is used, which were prepared by Kretzschmar et al. [4]. The convergence of the calculated quantities temperature and sound velocity at state 2 after 15 iteration steps was shown in [11]. The calculation of hydrogen or air was found at an inlet pressure 
of $875 \mathrm{bar}$ and an input temperature of $20^{\circ} \mathrm{C}$ and was determined using of an appropriate convergence criterion.

Using the continuity equation and the calculated state data in the narrowest cross-section, the mass flow is calculated for critical flow conditions and the conductance $\mathrm{C}$ according to ISO 6358 is determined with (23).

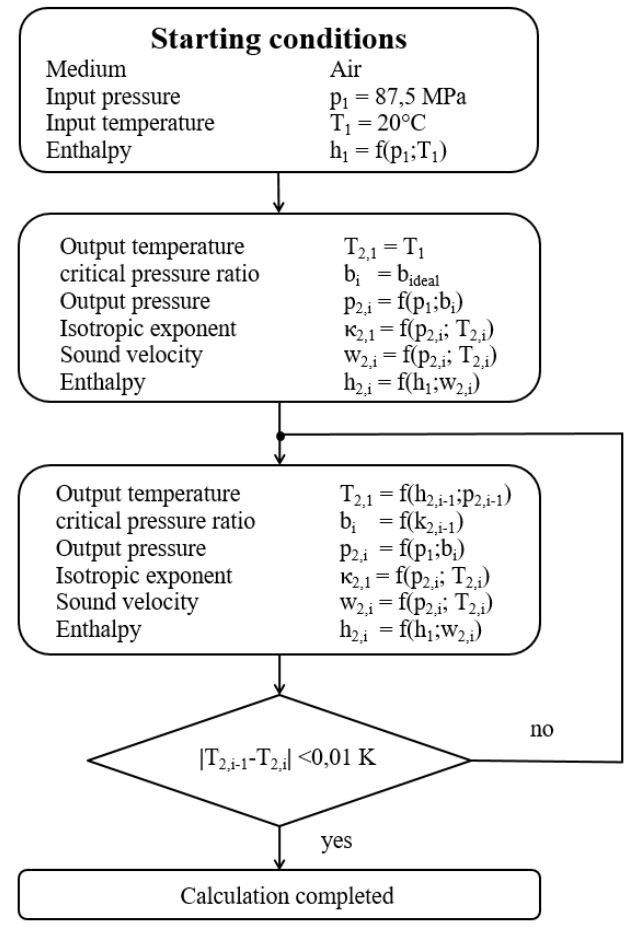

Figure 7: Flow chart for iterative state calculation in the narrowest cross section (all quantities were calculated with the equation of state of real gases; the index "real" was omitted for clarity)

The iteratively, with the equation of state of real gases, calculated parameter $\mathrm{C}$ is called $\mathrm{C}_{\text {real }}$ to distinguish it from the measured values. Using the same procedure, the parameters were determined at low pressure, i.e. with the equation of state of ideal gases, and is called the $\mathrm{C}_{\text {ideal }}$. The influence of the real gas properties on the conductance C, Fig. 8, can be described as follows (31).

$$
100 \cdot \frac{C_{\text {real }}-C_{\text {ideal }}}{C_{\text {real }}}
$$

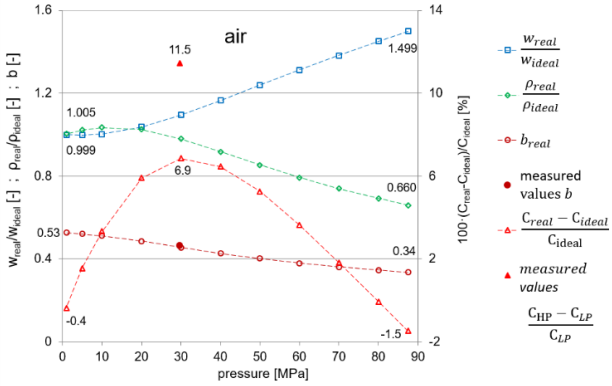

Figure 8: Iterative calculation of the state variables in the narrowest cross section. (assumptions: isentropic nozzle flow with real gas air)

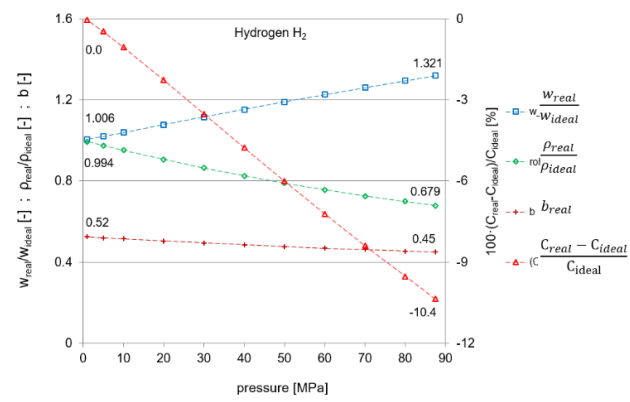

Figure 9: Iterative calculation of the state variables in the narrowest cross section. (assumptions: isentropic nozzle flow with real gas hydrogen)

An advantage of the iterative calculation of the thermodynamic state variables with the real gas data in the narrowest cross section is that the critical pressure ratio, the media density, the sound velocity and the pressure are calculated results. The process with increasing inlet pressure of the throttling shows, that the speed of sound increases significantly while the density decreases, see Fig. 8. These two properties have opposite effects on the mass flow, but they largely compensate each other. The critical pressure ratio measured with the geometry "nozzle" NW $0.5 \mathrm{~mm}$ is at $\mathrm{b}_{\mathrm{HP}}=0.463$, Tab. 2, and deviates only $1.5 \%$ from the calculated value at $\mathrm{p}_{1}=300 \mathrm{bar}$. This agreement results from the fact that for this geometry the assumption of lossfree flow is close to reality. The coefficient from equation (31) shows that the conductance $\mathrm{C}_{\text {real }}$ increases about $7 \%$ compared to $\mathrm{C}_{\text {ideal }}$ when flowing through a throttle at the pressure of 
300 bar, thus the mass flow increases at the same rate. With a further pressure increase $>300 \mathrm{bar}$, the deviation between $\mathrm{C}_{\text {ideal }}$ and $\mathrm{C}_{\text {real }}$ decreases again, as the effects of density and sound velocity on the mass flow are increasingly compensated. At $\mathrm{p}_{1}=800$ bar, the two coefficients are almost identical, but this does not mean that the gas behaves almost ideally. This is because the deviations in the speed of sound and density show that there is clearly real gas behavior in state 2 . Figure 8 shows the difference between the measured and calculated conductance ratio according to (31). Differences exist is because the measured pneumatic conductance values contain the flow-reducing effects, in contrast to the calculated conductance values. The calculated coefficient according to (31) in Fig. 8 on the other hand only shows the change due to the real gas properties with increasing pressure. To enable conversion of LP parameters, the flow-reducing effects both at low pressure and at high pressure must be known. If this is not the case, at least the influence of the real gas properties with the help of iterative calculation can be compensated.

The real gas properties strongly depend on the medium and accordingly, the effects on the mass flow through a throttle. The iterative calculation for the hydrogen medium in Fig. 9 shows, that unlike air, no increase in mass flow can be expected from real gas. On the contrary the conductivity at an inlet pressure of $\mathrm{p}_{1}=875 \mathrm{bar}$ and an input temperature of $\mathrm{T}_{1}=20^{\circ} \mathrm{C}$ is reduced by more than $10 \%$ in the comparison with the conductance of the ideal gas. The comparison between air and hydrogen under neglect of the flow-reducing effects also shows that the media properties have a significant influence on the critical pressure ratio. An isentropic change of the state starting at $\mathrm{p}_{1}=875$ bar leads to different thermodynamic states for air and hydrogen in the narrowest cross section.

The proposed iterative calculation of the state data in the narrowest cross section confirms the local validity of the parameters. A significant proportion of the measured differences in the conductance can be explained by the changed gas properties in the real state range. It was neglected that the flow-reducing effects can change at high pressure, whereby an exact conversion of lowpressure parameters and their application in the high-pressure range is made more difficult.

\section{SUMMARY}

The model proposed in ISO 6358 for the description of a stationary mass flow through a throttle is very well suited for use in the real gas sector, since the losses in parameters $\mathrm{C}$ and $\mathrm{b}$ are completely included. A mathematical description of the mass flow in the supercritical and subcritical range is thus given. The procedure for determining the model parameters $\mathrm{C}$ and $\mathrm{b}$ can therefore be derived without restrictions from ISO 6358, whereby the appropriate high pressure must be used for inlet pressure. The existing model becomes the model for real gases by using the parameters $\mathrm{C}_{\mathrm{HP}}$ and $\mathrm{b}_{\mathrm{HP}}$. The high-pressure parameters $\mathrm{C}_{\mathrm{HP}}$ and $\mathrm{b}_{\mathrm{HP}}$ of air at a pressure of 300 bar lead to low relative deviations of maximum $4.4 \%$ and average $1.0 \%$. Because of the good agreement between calculated and measured mass flow using the model, this procedure is also suitable for the simulation of components where gases at high pressure and real gas properties are used.

\section{NOMENCLATURE}

A $\quad\left[\mathrm{m}^{2}\right]$ Area cross-section

b [-] Critical compression ratio of pneumatic throttle valve [3]

C $\left[\mathrm{s} \cdot \mathrm{m}^{4} / \mathrm{kg}\right]$ Sonic conductance of pneumatic throttle valve

c $\quad[\mathrm{kJ} / \mathrm{kg} \cdot \mathrm{K}]$ Specific heat capacity

$h \quad[\mathrm{~J} / \mathrm{kg}]$ specific enthalpy

$\dot{m} \quad[\mathrm{~kg} / \mathrm{s}]$ mass flow

$p \quad[\mathrm{~Pa}]$ pressure

$R \quad[\mathrm{~J} / \mathrm{kg} \mathrm{K}]$ specific gas constant

$T \quad[\mathrm{~K}]$ temperature

$\Psi \quad[-]$ outflow function

$w \quad[\mathrm{~m} / \mathrm{s}]$ velocity

t. r. From test result

$Z \quad$ [-] Compressibility factor

$\alpha \quad$ [-] Flow contraction value

$\varepsilon \quad[-]$ Pressure ratio $\mathrm{p}_{2} / \mathrm{p}_{1}$

$\kappa \quad[-]$ Isentropic exponent

$\rho \quad\left[\mathrm{kg} / \mathrm{m}^{3}\right]$ mass density

$\varphi \quad[-]$ Speed characteristic number

$\Delta \dot{m} / \dot{m}[\%]$ relative deviation between computed and

$\cdot 10^{2}$ measured mass flow 


\section{INDEX}

$0 \quad$ Reference condition $\mathrm{p}=10^{5} \mathrm{~Pa}$ and $\mathrm{T}=293.15 \mathrm{~K}$ (International Organization for Standardization, International Standard; ISO 6358: pneumatic fluid power - components using compressible fluids - determination of flow-rate characteristics, 1989)

1 State forward throttle valve

2 State in the flow area from the throttle valve

* Supercritical flow condition $\left(\mathrm{w}=\mathrm{w}_{\mathrm{s}}\right.$ velocity of sound an $\mathrm{p}_{2} / \mathrm{p}_{1} \leq \mathrm{b}$ )

$H P \quad$ High pressure - identification parameter values $\mathrm{C}$ and $\mathrm{b}$ in the high-pressure range of real gas state

I Index for iterative calculation of the state in the flow area

ideal Computed value based on ideal gas law

$j \quad$ Index for the point of measurements for parameter identification

calc. Calculated values

crit. Critical flow condition $\left(\mathrm{w}=\mathrm{w}_{\mathrm{s}}\right.$ velocity of sound an $\mathrm{p}_{2} / \mathrm{p}_{1}=\mathrm{b}$ )

$L P \quad$ Low pressure - parameter identification values $\mathrm{C}$ and $\mathrm{b}$ in the low-pressure range with ideal gas law

NW Nominal diameter

max. Maximum values

meas. Measured values

real Based on equation of state real gas

$p \quad$ Isobaric

$v \quad$ isochoric

\section{REFERENCES}

[1] Deutsches Institut für Normung e. V. (2004) Deutsche Norm DIN EN ISO 4126-1: Sicherheitseinrichtungen gegen unzulässigen Überdruck - Teil 1: Sicherheitsventile

[2] Haak S (1991) Theoretische und experimentelle Untersuchungen zur Aussagefähigkeit strömungstechnischer Kenngrößen an ausgewählten Strukturen pneumatischer Steuerventile. Dissertation, Hochschule Erfurt

[3] International Organization for Standardization (1989) International Standard ISO 6358: pneumatic fluid power - components using compressible fluids - determination of flow-rate characteristics

[4] Kretzschmar H-J, Stöcker I, Jähne I, Kunick M (2008) Stoffwertprogramm, Bibliothek für Wasserstoff - H2. Hochschule Zittau/ Görlitz - University of Applied Sciences, Fachbereich Maschinenwesen, Fachgebiet Technische Thermodynamik

[5] Kretzschmar H-J, Stöcker I, von Dolsperg GD, Kunick M (2009) Property library for standard dry air. Hochschule Zittau/Görlitz -
University of Applied Sciences, Faculty of Mechanical Engineering, Department of Technical Thermodynamics

[6] Lemmon EW, Jacobsen RT, Penoncello SG, Friend DG (2000) Thermodynamic properties of air and mixtures of nitrogen, argon, and oxygen from 60 to $2000 \mathrm{~K}$ at pressures to 2000 MPa. J Phys Chem Ref Data 29(3):331-385

[7] Leachman JW, Jacobsen RT, Penoncello SG, Lemmon EW (2009) Fundamental equations of state for parahydrogen, normal hydrogen, and orthohydrogen. J Phys Chem Ref Data 38(3):721-748

[8] Murrenhoff H (2006) Grundlagen der Fluidtechnik, Teil 2: Pneumatik, 2 Aufl. Shaker Verlag, Aachen

[9] Sanville FE (1971) A new method of specifying the flow capacity of pneumatic fluid power valves. Second Fluid Power Symposium 4th-7th Jan. Guildford, S D3-37D3-47

[10] Schmidt J, Peschel W, Beune A (2009) Experimentelle und theoretische Untersuchungen zu Hochdruck-Sicherheitsventilen - Auslegung und Design unterstützt durch numerische Rechenmethoden (CFD). Forschung im Ingenieurwesen 73:105-117

[11] Ramsperger M, Pasieka L (2014) Zur Anwendwendbarkeit des Massenstrom-Modells nach ISO 6358 mit den Kennwerten Leitwert $\mathrm{C}$ und kritisches Druckverhältnis b für Gase im Hochdruckbereich. Springer, Forsch Ingenieurwes DOI 10.1007/s10010-014-0177-7

[12] International Organization for Standardization (2013) International Standard ISO 6358-1: Determination of flow-rate characteristics of components using compressible fluids - Part 1: General rules and test methods for steady-state flow 